\title{
Stereotactic Radiosurgery for Recursive Partitioning Analysis Class II / III Lung Cancer Patients with Brain Metastases in the Modern Treatment Era
}

In Bong Ha ${ }^{1}$, Jin Ho Song ${ }^{2,3}$, Bae Kwon Jeong ${ }^{1,3}$, Hojin Jeong ${ }^{1,3}$, Yun Hee Lee ${ }^{1,3}$, Hoon Sik Choi ${ }^{2}$ and Ki Mun Kang 2,3,*

${ }^{1}$ Department of Radiation Oncology, Gyeongsang National University School of Medicine, and Gyeongsang National University Hospital, Jinju, Korea

${ }^{2}$ Department of Radiation Oncology, Gyeongsang National University School of Medicine, and Gyeongsang National University Changwon Hospital, Changwon, Korea

${ }^{3}$ Institute of Health Science, Gyeongsang National University, Jinju, Korea

* Author to whom correspondence should be addressed.

Address for correspondence:

Ki Mun Kang, MD, PhD

Department of Radiation Oncology, Gyeongsang National University Changwon Hospital,

13 Samjungja-ro, Changwon 51472, Republic of Korea

E-mail: jsk92@.gnu.ac.kr 
Abstract: Stereotactic radiosurgery (SRS) is considered the initial treatment for lung cancer patients with small-sized and limited number of brain metastases. The objective of this study was to assess clinical outcomes of SRS treatment using CyberKnife (CK) for recursive partitioning analysis (RPA) class II/III patients with one to three brain metastases from lung cancer and identify which patients in the high RPA class could benefit from SRS. A total of 48 lung cancer patients who received CK-based SRS for their metastatic brain lesions from 2010 to 2017 were retrospectively analyzed. Radiographic response was evaluated during follow-up period. Overall survival (OS) and intracranial progressionfree survival (IPFS) were calculated and prognostic variables associated with OS and IPFS were evaluated. Median follow-up time was 6.6 months. Local control rates at 6 months and 1-year following SRS were $98 \%$ and $92 \%$, respectively. The median OS of all patients was 8 months. One-year and 2year OS rates were $40.8 \%$ and $20.9 \%$, respectively. In multivariate analysis, uncontrolled primary disease $(p=0.008)$ and ECOG performance status of 2 or $3(p=0.001)$ were independent prognostic factors for inferior OS. These two factors were also significantly associated with inferior IPFS. In subgroup analysis according to RPA class, primary disease status was the only prognostic factor, showing statistically significant OS differences in both RPA class II and III (controlled vs. uncontrolled: 41.1 vs. 12.3 months in RPA class II, $p=0.031 ; 26.9$ vs. 4.1 months in RPA class III, $p=0.011$ ). Our results indicated that SRS could be an effective treatment option for RPA class II/III patients with brain metastases from lung cancer in the modern treatment era. SRS might be particularly considered for patients with controlled primary disease.

Keywords: lung cancer; brain metastase; prognostic factors; stereotactic radiosurgery 


\section{Introduction}

Lung cancer has currently the highest incidence in the world. It is the leading cause of cancer related deaths [1]. Brain metastases from lung cancer also have a high incidence rate of $20 \%$ [2]. As this disease progresses, brain metastases occur more often. Among all cases of brain metastases, those from lung cancer account for $40-50 \%$ [3]. Therefore, appropriate management of brain metastases is a very important issue in treatment of lung cancer patients.

In the last several decades, whole brain radiation therapy (WBRT) is a standard treatment for patients with multiple brain metastases. However, many clinical studies have shown that the quality of life of patients is deteriorated by the neurotoxicity caused by WBRT $[3,4]$. Although median survival of patients with brain metastases treated with WBRT has been reported to be less than 6 months, the number of patients with long term survival as well as median survival of patients has increased recently due to the development of systemic therapies and choice of appropriate local treatment strategies, e.g., SRS alone, SRS plus WBRT and surgical resection. $[5,6]$. Treatment for four or fewer oliogometastatic lesions has been gradually replaced by SRS or surgical resection, instead of WBRT which reduces neurotoxicity while not affect survival. [7,8]. In addition, some studies have reported that SRS alone is an effective treatment for patients with five or more brain metastases [9-11].

When determining whether patients with brain metastases should receive radical local treatment, recursive partitioning analysis (RPA) classification has been widely used [5]. Although it is a readily available prognostic tool to select candidates, it lacks other important prognostic factors such as histology of tumor, number of brain metastases, and molecular features of tumor. [3,12,13]. To perform treatment for brain metastases based on individual assessment of prognostic factors, it is necessary to consider all significant prognostic factors for survival. Considering median survival of patients with brain metastases, it is desirable to try aggressive treatment strategy for RPA class I patients. However, the number of such patients is very limited because most patients belong to class II and III in clinical 
practice [14]. Although some studies have reported SRS results in lung cancer patients with limited number of brain metastases, few studies have reported the treatment outcomes of SRS for patients with only RPA class II/III.

Thus, the aim of this study was to analyze clinical outcomes of SRS using CyberKnife (Accuray Inc., Snnyvale, CA, USA) for RPA class II/III patients with one to three brain metastases from lung cancer and identify which patients in the high RPA class could benefit from SRS.

\section{Results}

\subsection{Patient and tumor characteristics}

The median age of the 48 patients analyzed in this study was 68.5 years (range, $48-82$ years). The median follow-up time was 6.6 months (range, 0.6-89.7 months). Of these 48 patients, 37 (77.1\%) were previously diagnosed with non-small cell lung cancer (NSCLC) and $11(22.9 \%)$ were diagnosed with SCLC. Of these NSCLC patients, 23, 9, 2, and 3 patients were diagnosed with adenocarcinoma, squamous cell carcinoma, large cell carcinoma, and 'not otherwise specified', respectively. The Eastern Cooperative Oncology Group (ECOG) performance status was 0-1 in 28 (58.3\%) patients and 2-3 in 20 (41.7\%) patients. At the time of the initial radiosurgery, 9 (18.8\%) patients were asymptomatic, 26 (54.2\%) patients had headache, $9(18.8 \%)$ patients had unilateral weakness, $6(12.5 \%)$ patients had seizure, $4(8.3 \%)$ patients had dysarthria, and $2(4.2 \%)$ patients had ataxia. Ten (20.8\%) patients had uncontrolled primary disease and $31(64.6 \%)$ patients had extracranial metastases. Twenty-eight (58.3\%) and $20(41.7 \%)$ patients were classified as RPA class II and III, respectively. Of the total of 48 patients, 20 (41.7\%) previously received WBRT, including five patients with small cell lung cancer (SCLC) who underwent prophylactic cranial irradiation (PCI). Nine of these 20 patients who received WBRT underwent SRS for salvage treatment for recurrent brain metastases while the other 11 patients underwent SRS as boost treatment for WBRT. Planning target size ranged from 0.5 to $5.0 \mathrm{~cm}$ (median 
$2.0 \mathrm{~cm}$ ) and target volume ranged from 0.2 to $78.9 \mathrm{ml}$ (median $2.6 \mathrm{ml}$ ). Patient and tumor characteristics are detailed in Table 1.

\subsection{Treatment outcomes}

LC rates at 6 months and 1-year following SRS were 98\% and 92\%, respectively. A representative case of good response after CK-based SRS for brain lesion is shown in Figure 1. RC rates at 6 months and 1-year following SRS were $88 \%$ and $78 \%$, respectively. SRS site failure occurred in five patients while distant brain failure occurred in 12 patients during the follow-up period after SRS.

At the time of analysis, $41(85.4 \%)$ patients died, including $26(54.2 \%)$ patients due to cancer progression, $4(8.3 \%)$ due to intracranial progression, $2(4.2 \%)$ due to non-cancerous cause, and 9 (18.8\%) due to unknown reason. The median OS of all patients was 8 months. One-year and 2-year OS rates were $40.8 \%$ and $20.9 \%$, respectively (Fig. 2A). In univariate analysis for OS, primary disease status (controlled vs. uncontrolled: 31 vs. 5.6 months; $p=0.006)$, ECOG performance status (0-1 vs. 23: 12.3 vs. 4.2 months; $p=0.007$ ), extracranial metastases (absent vs. present: 17.3 vs. 5.6 months; $p=$ 0.023 ), and primary histology (NSCLC vs. SCLC: 8.5 vs. 3.6 months; $p=0.024$ ) were significant prognostic factors (Table 2). Multivariate analysis showed that uncontrolled primary disease $(p=0.008)$ and ECOG performance status of 2-3 $(p=0.001)$ were independent prognostic factors for inferior OS (Table 3).

The median IPFS of all patients was 5.3 months. One-year and 2-year IPFS rates were $23.9 \%$ and 15.2\%, respectively (Fig. 2B). Similar to the analysis for OS, primary disease status and ECOG performance status were significant prognostic factors for IPFS (Table 3).

We also analyzed OS according to primary disease status and the presence of extracranial metastases in each RPA class II and III group. In RPA class II group, median OS time of patients with controlled primary disease was significantly higher than that of patients with uncontrolled primary disease (41.1 vs. 12.3 months; $p=0.031$; Fig. 3A). Patients without extracranial metastases had significantly higher median OS than those with extracranial metastases (29.1 vs. 8.5 months; $p=0.032$; Fig. 3B). In the 
RPA class III group, only OS according to primary disease status showed statistically significant difference (controlled vs. uncontrolled: 26.9 vs. 4.1 months; $p=0.011$; Fig. $4 \mathrm{~A}$ ). OS according to the presence of extracranial metastases also showed a large difference of 5.1 months (absent vs. present: 9.3 vs. 4.2 months; $p=0.072$; Fig. 4B), although the statistical significance was marginal.

No symptoms or signs caused by radiation induced necrosis were observed during follow-up period after SRS. In addition, there was no case showing significant neurocognitive dysfunction. Other complications included seizure in two patients and IICP signs in three patients due to cerebral edema.

\section{Discussion}

Recently, with the advent of novel targeted or immunotherapeutic agents, rapid development of systemic therapy has improved survival and clinical outcomes of patients with metastatic lung cancer [15-20]. Previously, SRS for brain metastases is known to be an effective alternative treatment modality for WBRT in patients with RPA class I predicted to have good prognosis [5,21]. There are not so many clinical studies on patients with RPA class II / III who are predicted to have poor prognosis. Particularly RPA class III patients were excluded from most randomized studies because of their extremely poor prognosis $[22,23]$. However, in the modern treatment era, when chemotherapeutic agents with acceptable toxicity and advanced supportive management are introduced, lung cancer patients with brain metastases not necessarily have lower survival rates due to old age or poor performance [24,25]. We have conducted CK-based SRS since 2010, which is the time period after application of targeted therapies such as epidermal growth factor receptor tyrosine kinase inhibitors to stage IV lung cancer in our institution. Our results showed that, with the help of advanced systemic therapies, survival benefit can be achieved through aggressive local treatment for brain lesions if primary disease is adequately controlled.

Since the publication of RPA classification system by Gaspar et al., it has been questionable whether this could be generally applied to determine the treatment strategies of patients with brain metastases 
[5]. A few studies have been conducted to verify this system [14,26,27]. Nieder et al. have confirmed that patients with RPA class I have no disagreement with aggressive local treatment such as SRS [14]. However, considering time to non-CNS death, primary disease controlled subgroups of RPA class II patients suggest that aggressive local control of brain metastases may provide survival benefit [14]. Yamamoto et al. have divided RPA class II into three subclasses by scoring four prognostic factors (KPS, tumor numbers, primary disease status, and non-brain metastases) and reported that prognostic factors that can determine RPA class II are diverse and very heterogeneous, showing significant differences among subclasses for OS ( $p<0.001$ for all subclasses) [27]. In the above study, they included tumor numbers that could be associated with target volume rather than age, one of the prognostic factors that determine original RPA class, suggesting the importance of local control in the era of advanced systemic therapies [27].

One retrospective study has shown that RPA class III patients with brain metastases have a reasonable median OS of 7.2 months when they are treated with SRS alone [25]. That study also argues that poor performance patients may be ideal candidates for SRS because low incidence of distant CNS failure during life expectancy and single faction treatment can be helpful for patients with debility and their caregivers [25]. We also believe that the convenience of short fractionation is a clear benefit for poor performance patients and SRS is a more effective treatment than WBRT in terms of the possibility of combination with chemotherapy or in the absence of delaying systemic therapy.

According to our study results, significant prognostic factors for both OS and IPFS in multivariate analysis were primary disease status and ECOG performance status. Primary disease status has been previously identified as the strongest prognostic factor associated with survival and intracranial progression in several studies $[12,28,29]$. This plays a very important role in clinical decision-making, in predicting long term outcome after treatment of patients with brain metastases, and when using treatment strategies such as upfront SRS. The ECOG scale is the simplest and most commonly used performance status scale in clinical practice. It was used in our study. Our study only included patients 
with RPA class II and III. The factor that determines these two classes is only Karnofsky performance status (KPS). When assessing the clinical performance status of patients, patient's ability to perform certain activities of daily living without help of others is regarded as up to 1 according to the ECOG scale and up to 70 according to KPS. We excluded RPA class from the analysis because groups categorized by ECOG (0-1 vs 2) were perfectly matched with groups categorized by RPA class ( 2 vs 3 ). This shows that the RPA class also serves as a statistically significant prognostic factor for survival. The presence of extracranial metastases is known to be a significant poor prognostic factor for survival in several clinical studies, including factors that should be considered in diagnosis-specific graded prognostic assessment (DS-GPA) by stratifying based on primary cancer histology as well as RPA classification for brain metastases [5,13,21]. The presence of extracranial metastases in our study was a significant prognostic factor in univariate analysis of OS and IPFS. However, it did not show any statistically significant difference in multivariate analysis. Thus, primary disease status, performance status, and extracranial metastases are important factors that have a significant effect on OS and IPFS. In each group of RPA class II and III, we further analyzed the OS for these two factors (primary disease status, extracranial metastases) showing significantly survival difference in univariate analysis except for age among the three factors determining RPA class II. As a result, controlled primary disease was associated with significantly superior OS in both RPA class II and III. The absence of extracranial metastases was also associated with significantly superior OS in RPA class II. Particularly, RPA class III patients with poor performance had a median survival time longer than 2 years if the primary disease was controlled. These results clearly suggest that some RPA class II/III patients can have good prognosis depending on the control of primary tumor and extracranial metastatic lesions in the modern treatment era.

When we identified adverse effects, there were no patients with significant neurocognitive dysfunction or radionecrosis. Only $10 \%$ of patients suffered from neurologic toxicities after SRS. Side effects might 
have been underestimated due to evaluations without a quantitative neurocognitive test. Individual setting of dose schedules at clinician's discretion might have also affected the severity of side effects.

We acknowledge that this retrospective study has several limitations including selection bias and confounding factors. In particular, patients who previously had WBRT were included. Heterogeneous lung cancer group was formed by including both SCLC and NSCLC patients. In addition, the relatively small sample size limits the statistical power. Thus, further studies with a large number of patients are needed to validate our findings. In particular, a well-designed prospective study will help determine which patients will need active local treatment or just supportive care.

\section{Material and methods}

\subsection{Patient selection}

This retrospective study was approved by Institutional Review Board (IRB) of Gyengsang National University Hospital (IRB number: 2018-05-015).

A total of 106 patients with brain metastases were treated with SRS using CyberKnife (CK) at Gyengsang National University Hospital between February 2010 and May 2017. Among them, patients with primary lung cancer who had completed planned schedule of SRS were included while patients with RPA class I or loss of follow-up were excluded. Finally, 48 lung cancer patients eligible for RPA class II / III were retrospectively analyzed. SRS was performed in patients with less than three metastatic brain lesions. The maximum diameter of each lesion was less than $5 \mathrm{~cm}$ according to our institutional protocol.

\subsection{SRS}

All patients underwent SRS using CK. During treatments, all patients were immobilized with a thermoplastic head mask in supine position. Contrast-enhanced computed tomography (CT) and contrast-enhanced magnetic resonance imaging (MRI) for brain were taken with slice thickness of 1.0 $\mathrm{mm}$ and $2.5 \mathrm{~mm}$, respectively. CT and MRI images were then rigidly fused with respect to each other 
using CK planning system (Multiplan ver. 3.5.4). Gross tumor volume (GTV) was defined as enhanced lesion observed by any of both images. First, GTV was delineated on a CT image. It was then modified to include clearly contrast-enhancing regions seen in MRI images. Clinical target volume (CTV) was set to the same as GTV, assuming no microscopic invasion outside the GTV. Planning target volume (PTV) was generated by adding 1-mm margin from the GTV. Organs at risk (OAR) including lenses, optic nerves, optic chasm, brainstem, and spinal cord were also contoured. If two lesions were close to $1 \mathrm{~cm}$ or less, they were planned as one target. For this reason, although the number of brain metastatic lesions in all patients treated was 70 , the actual number of planning targets was 63 . Inverse treatment planning was performed for all patients using Multiplan version 3.5.4 (Accuray Inc., Snnyvale, CA, USA). SRS treatment plans were basically designed so that the entire GTV and at least 95\% PTV were covered by the prescription dose surface. The prescription dose was normalized median $80 \%$ isodose line (range, $75 \%$ to $85 \%$ ) relative to the maximal dose. The prescription dose was basically determined based on volume-dependent dose regimen suggested in the Radiation Therapy Oncology Group (RTOG) 95-05 trial [5]. It was partly revised according to the judgment of clinicians considering the tumor size and location, the timing of SRS, and prior RT dose. Details in the prescription dose actually delivered are summarized in the Table 4 along with dose schedules and biologic equivalence dose for $\alpha / \beta=10$ $\left(\mathrm{BED}_{10}\right)$.

\subsection{Response and outcome assessment}

After SRS, follow-up MRI (or CT if ineligible for MRI) was performed every 2 to 3 months, or when clinically indicated. Tumor response was evaluated based on Response Evaluation Criteria in Solid Tumors (RECIST) criteria (version 1.1) [30]. In the case of multiple lesions, the sum of the two largest lesions was used to evaluate the response. Complete response, partial response, and stable disease were classified as local control (LC) while progressive disease was classified as local failure.

Overall survival (OS), intracranial progression-free survival (IPFS), LC, and regional control (RC) were evaluated. OS was defined as the length of time from the date of diagnosis of brain metastases to death 
or the last follow-up. IPFS was defined as the length of time from the date of diagnosis of brain metastases to intracranial progression or death from any cause or the last follow-up. However, in the case of salvage SRS, the survival time was calculated from the time of diagnosis of recurrent brain metastases in MRI. LC was defined as freedom from development of new lesions within the field treated with SRS or progression in preexisting metastases. RC was defined as freedom from development of new distant brain metastases.

Adverse effects such as increased intracranial pressure (IICP) signs, neurocognitive defect, and radiation necrosis were also evaluated. Quantitative evaluation such as questionnaire on neurocognitive function was not made. However, a clear clinical record of patient's symptoms was obtained through a simple clinical interview with the clinician.

\subsection{Statistical analysis}

All statistical analyses were performed using SPSS software Version 21.0 for Windows. Actuarial OS, IPFS, LC rates, and RC rates were calculated using the Kaplan-Meier method. Log-rank test was used for univariate analysis to assess prognostic factors associated with OS and IPFS. Cox proportional hazard models were performed for multivariate analysis. A two-sided $p$-value $<0.05$ was considered statistically significant.

\section{Conclusions}

In conclusion, our results showed that SRS could be a useful treatment modality for RPA class II/III patients with one to three brain metastases from lung cancer in the modern treatment era. Our results suggest that patients with older age or poor performance should not be unconditionally excluded from aggressive treatment for brain metastases and SRS might be considered as an initial treatment for RPA class II/III patients with well-controlled primary disease.

Acknowledgments: This study was not funded by any organizations or companies. 
Author Contributions: Conceptualization, I.B.H. and K.M.K.; Date curation, I.B.H. and B.K.J.; Formal analysis, I.B.H., B.K.J. and K.M.K.; Funding acquisition, K.M.K.; Investigation, I.B.H., H.J. and H.S.C.; Methodology, H.J. and Y.H.L.; Project administration, J.H.S., Y.H.L. and H.S.C.; Resources, Y.H.L. and H.S.C.; Software, I.B.H. and Y.H.L.; Supervision, K.M.K.; Validation, J.H.S., H.J., H.S.C. and K.M.K.; Visualization, I.B.H.; Writing - original draft, I.B.H. and K.M.K; Writing - review \& editing, I.B.H. and K.M.K.

Conflicts of Interest: The authors declare no conflict of interest.

\section{References}

1. Torre, L.A.; Bray, F.; Siegel, R.L.; Ferlay, J.; Lortet-Tieulent, J.; Jemal, A. Global cancer statistics, 2012. CA: a cancer journal for clinicians 2015, 65, 87-108.

2. Barnholtz-Sloan, J.S.; Sloan, A.E.; Davis, F.G.; Vigneau, F.D.; Lai, P.; Sawaya, R.E. Incidence proportions of brain metastases in patients diagnosed (1973 to 2001) in the metropolitan detroit cancer surveillance system. Journal of clinical oncology : official journal of the American Society of Clinical Oncology 2004, 22, 2865-2872.

3. Jezierska, D.; Adamska, K.; Liebert, W. Evaluation of results of linac-based radiosurgery for brain metastases from primary lung cancer. Reports of practical oncology and radiotherapy: journal of Greatpoland Cancer Center in Poznan and Polish Society of Radiation Oncology 2014, 19, 19-29.

4. $\quad$ Aoyama, H.; Tago, M.; Kato, N.; Toyoda, T.; Kenjyo, M.; Hirota, S.; Shioura, H.; Inomata, T.; Kunieda, E.; Hayakawa, K., et al. Neurocognitive function of patients with brain metastasis who received either whole brain radiotherapy plus stereotactic radiosurgery or radiosurgery alone. International journal of radiation oncology, biology, physics 2007, 68, 1388-1395.

5. Gaspar, L.; Scott, C.; Rotman, M.; Asbell, S.; Phillips, T.; Wasserman, T.; McKenna, W.G.; Byhardt, R. Recursive partitioning analysis (rpa) of prognostic factors in three radiation therapy oncology group (rtog) brain metastases trials. International journal of radiation oncology, biology, physics 1997, 37, 745-751.

6. Tabouret, E.; Chinot, O.; Metellus, P.; Tallet, A.; Viens, P.; Goncalves, A. Recent trends in epidemiology of brain metastases: An overview. Anticancer research 2012, 32, 4655-4662.

7. Mut, M. Surgical treatment of brain metastasis: A review. Clinical neurology and neurosurgery 2012, 114, 1-8.

8. Gupta, T. Stereotactic radiosurgery for brain oligometastases: Good for some, better for all? Annals of oncology : official journal of the European Society for Medical Oncology 2005, 16, 1749-1754.

9. Hunter, G.K.; Suh, J.H.; Reuther, A.M.; Vogelbaum, M.A.; Barnett, G.H.; Angelov, L.; Weil, R.J.; Neyman, G.; Chao, S.T. Treatment of five or more brain metastases with stereotactic radiosurgery. International journal of radiation oncology, biology, physics 2012, 83, 1394-1398.

10. Yamamoto, M.; Kawabe, T.; Sato, Y.; Higuchi, Y.; Nariai, T.; Watanabe, S.; Kasuya, H. Stereotactic radiosurgery for patients with multiple brain metastases: A case-matched study comparing treatment results for patients with 2-9 versus 10 or more tumors. Journal of neurosurgery 2014, 121 Suppl, 16-25. 
11. Zindler, J.D.; Slotman, B.J.; Lagerwaard, F.J. Patterns of distant brain recurrences after radiosurgery alone for newly diagnosed brain metastases: Implications for salvage therapy. Radiotherapy and oncology : journal of the European Society for Therapeutic Radiology and Oncology 2014, 112, 212-216.

12. Gorovets, D.; Ayala-Peacock, D.; Tybor, D.J.; Rava, P.; Ebner, D.; Cielo, D.; Noren, G.; Wazer, D.E.; Chan, M.; Hepel, J.T. Multi-institutional nomogram predicting survival free from salvage whole brain radiation after radiosurgery in patients with brain metastases. International journal of radiation oncology, biology, physics 2017, 97, 246-253.

13. Sperduto, P.W.; Kased, N.; Roberge, D.; Xu, Z.; Shanley, R.; Luo, X.; Sneed, P.K.; Chao, S.T.; Weil, R.J.; Suh, J., et al. Summary report on the graded prognostic assessment: An accurate and facile diagnosis-specific tool to estimate survival for patients with brain metastases. Journal of clinical oncology : official journal of the American Society of Clinical Oncology 2012, 30, 419425.

14. Nieder, C.; Nestle, U.; Motaref, B.; Walter, K.; Niewald, M.; Schnabel, K. Prognostic factors in brain metastases: Should patients be selected for aggressive treatment according to recursive partitioning analysis (rpa) classes? International journal of radiation oncology, biology, physics 2000, 46, 297-302.

15. Pilkington, G.; Boland, A.; Brown, T.; Oyee, J.; Bagust, A.; Dickson, R. A systematic review of the clinical effectiveness of first-line chemotherapy for adult patients with locally advanced or metastatic non-small cell lung cancer. Thorax 2015, 70, 359-367.

16. Yang, W.C.; Xiao, F.; Shih, J.Y.; Ho, C.C.; Chen, Y.F.; Tseng, H.M.; Chen, K.Y.; Liao, W.Y.; Yu, C.J.; Yang, J.C., et al. Epidermal growth factor receptor mutation predicts favorable outcomes in non-small cell lung cancer patients with brain metastases treated with stereotactic radiosurgery. Radiotherapy and oncology : journal of the European Society for Therapeutic Radiology and Oncology 2018, 126, 368-374.

17. Nieder, C.; Hintz, M.; Oehlke, O.; Bilger, A.; Grosu, A.L. Validation of the graded prognostic assessment for lung cancer with brain metastases using molecular markers (lung-molgpa). Radiation oncology 2017, 12, 107.

18. Brahmer, J.; Reckamp, K.L.; Baas, P.; Crino, L.; Eberhardt, W.E.; Poddubskaya, E.; Antonia, S.; Pluzanski, A.; Vokes, E.E.; Holgado, E., et al. Nivolumab versus docetaxel in advanced squamous-cell non-small-cell lung cancer. The New England journal of medicine 2015, 373, 123-135.

19. Borghaei, H.; Paz-Ares, L.; Horn, L.; Spigel, D.R.; Steins, M.; Ready, N.E.; Chow, L.Q.; Vokes, E.E.; Felip, E.; Holgado, E., et al. Nivolumab versus docetaxel in advanced nonsquamous nonsmall-cell lung cancer. The New England journal of medicine 2015, 373, 1627-1639.

20. Goldberg, S.B.; Gettinger, S.N.; Mahajan, A.; Chiang, A.C.; Herbst, R.S.; Sznol, M.; Tsiouris, A.J.; Cohen, J.; Vortmeyer, A.; Jilaveanu, L., et al. Pembrolizumab for patients with melanoma or non-small-cell lung cancer and untreated brain metastases: Early analysis of a nonrandomised, open-label, phase 2 trial. The Lancet. Oncology 2016, 17, 976-983.

21. Gaspar, L.E.; Scott, C.; Murray, K.; Curran, W. Validation of the rtog recursive partitioning analysis (rpa) classification for brain metastases. International journal of radiation oncology, biology, physics 2000, 47, 1001-1006.

22. $\quad$ Chang, E.L.; Wefel, J.S.; Hess, K.R.; Allen, P.K.; Lang, F.F.; Kornguth, D.G.; Arbuckle, R.B.; Swint, J.M.; Shiu, A.S.; Maor, M.H., et al. Neurocognition in patients with brain metastases treated with radiosurgery or radiosurgery plus whole-brain irradiation: A randomised controlled trial. The Lancet. Oncology 2009, 10, 1037-1044.

23. Aoyama, H.; Shirato, H.; Tago, M.; Nakagawa, K.; Toyoda, T.; Hatano, K.; Kenjyo, M.; Oya, N.; Hirota, S.; Shioura, H., et al. Stereotactic radiosurgery plus whole-brain radiation therapy vs stereotactic radiosurgery alone for treatment of brain metastases: A randomized controlled trial. Jama 2006, 295, 2483-2491. 
24. Hanna, N.; Johnson, D.; Temin, S.; Baker, S., Jr.; Brahmer, J.; Ellis, P.M.; Giaccone, G.; Hesketh, P.J.; Jaiyesimi, I.; Leighl, N.B., et al. Systemic therapy for stage iv non-small-cell lung cancer: American society of clinical oncology clinical practice guideline update. Journal of clinical oncology : official journal of the American Society of Clinical Oncology 2017, 35, 3484-3515.

25. Kubicek, G.J.; Turtz, A.; Xue, J.; Patel, A.; Richards, G.; LaCouture, T.; Cappelli, L.; Diestelkamp, T.; Saraiya, P.; Bexon, A., et al. Stereotactic radiosurgery for poor performance status patients. International journal of radiation oncology, biology, physics 2016, 95, 956-959.

26. Nieder, C.; Andratschke, N.; Grosu, A.L.; Molls, M. Recursive partitioning analysis (rpa) class does not predict survival in patients with four or more brain metastases. Strahlentherapie und Onkologie : Organ der Deutschen Rontgengesellschaft ... [et al] 2003, 179, 16-20.

27. Yamamoto, M.; Sato, Y.; Serizawa, T.; Kawabe, T.; Higuchi, Y.; Nagano, O.; Barfod, B.E.; Ono, J.; Kasuya, H.; Urakawa, Y. Subclassification of recursive partitioning analysis class ii patients with brain metastases treated radiosurgically. International journal of radiation oncology, biology, physics 2012, 83, 1399-1405.

28. Tsao, M.; Xu, W.; Sahgal, A. A meta-analysis evaluating stereotactic radiosurgery, whole-brain radiotherapy, or both for patients presenting with a limited number of brain metastases. Cancer 2012, 118, 2486-2493.

29. Wegner, R.E.; Olson, A.C.; Kondziolka, D.; Niranjan, A.; Lundsford, L.D.; Flickinger, J.C. Stereotactic radiosurgery for patients with brain metastases from small cell lung cancer. International journal of radiation oncology, biology, physics 2011, 81, e21-27.

30. Eisenhauer, E.A.; Therasse, P.; Bogaerts, J.; Schwartz, L.H.; Sargent, D.; Ford, R.; Dancey, J.; Arbuck, S.; Gwyther, S.; Mooney, M., et al. New response evaluation criteria in solid tumours: Revised recist guideline (version 1.1). Eur J Cancer 2009, 45, 228-247. 


\section{Tables}

Table 1. Patient and tumor characteristics

\begin{tabular}{|c|c|c|}
\hline Variables & Number of & atients $(\%)$ \\
\hline \multicolumn{3}{|l|}{ Age (years) } \\
\hline Median (range) & 68.5 & $(48-82)$ \\
\hline$<65$ & 18 & $(37.5)$ \\
\hline$\geq 65$ & 30 & $(62.5)$ \\
\hline \multicolumn{3}{|l|}{ Gender } \\
\hline Male & 39 & $(81.3)$ \\
\hline Female & 9 & $(18.8)$ \\
\hline \multicolumn{3}{|l|}{ Histological type } \\
\hline NSCLC & 37 & $(77.1)$ \\
\hline SCLC & 11 & $(22.9)$ \\
\hline \multicolumn{3}{|c|}{ ECOG performance status } \\
\hline $0-1$ & 28 & $(58.3)$ \\
\hline $2-3$ & 20 & $(41.7)$ \\
\hline \multicolumn{3}{|l|}{ Neurologic status } \\
\hline Symptomatic & 39 & $(81.3)$ \\
\hline Asymptomatic & 9 & $(18.8)$ \\
\hline \multicolumn{3}{|c|}{ Primary disease status } \\
\hline Controlled & 10 & $(20.8)$ \\
\hline
\end{tabular}


Uncontrolled

Extracranial metastases

Present

Absent

RPA class

2

3

Number of brain lesions

1

2

3

Prior WBRT (include PCI)

No

Yes

Target size $(\mathrm{cm})$

Median (range)

Target volume (ml)

Median (range)
$38 \quad(79.2)$

31 (64.6)

$17 \quad(35.4)$

$28 \quad(58.3)$

$20 \quad(41.7)$

$35 \quad(72.9)$

$5 \quad(10.4)$

$8 \quad(16.7)$

$28 \quad(58.3)$

$20 \quad(41.7)$

$2.0 \quad(0.5-5.0)$

$2.6 \quad(0.2-78.9)$

NSCLC: Non-small cell lung cancer; SCLC: Small cell lung cancer; ECOG: Eastern Cooperative

Oncology group; RPA: Recursive partitioning analysis; WBRT: Whole brain radiation therapy; PCI:

Prophylactic cranial irradiation.

Table 2. Results of univariate analysis of overall survival and brain failure free survival 


\begin{tabular}{llllll}
\hline Variables & No. & Median OS & $P$-value & Median BFFS & $P$-value
\end{tabular}

(months)

(months)
Uncontrolled

ECOG performance status

$0-1$

$2-3$

Extracranial metastases

Absent

Present

Histological type

NSCLC

SCLC

No. of brain lesions

1

$\geq 2$

Neurologic status

Asymptomatic

Symptomatic

Age

$<65$

$\geq 65$

Prior WBRT

0.541

0.007

31

6.7

38

10

28

20

12.3

7.8

4.2

4.1

0.006

4.7

26.9

0.044

0.001

$$
17
$$

17.3

10.2

31

6.7

4.7

0.036

0.070

37

9.9

7.5

11

5.3

2.4

0.666

0.394

35

6.7

5.1

13

12.3

8.0

0.270

0.904

9

12.3

6.7

39

8.0

5.4

0.463

0.807

18

12.3

7.3

30

5.4

4.7

0.781 


$\begin{array}{llll}\text { No } & 28 & 7.5 & 6.7 \\ \text { Yes } & 20 & 8.5 & 5.1\end{array}$

No.: number; OS: overall survival; BFFS: brain failure free survival; ECOG: Eastern Cooperative Oncology group; NSCLC: Non-small cell lung cancer; SCLC: Small cell lung cancer; WBRT: Whole brain radiation therapy.

Table 3. Results of multivariate analysis of overall survival and brain failure free survival

\begin{tabular}{|c|c|c|c|c|c|c|}
\hline \multirow[t]{2}{*}{ Variables } & \multicolumn{3}{|l|}{ OS } & \multicolumn{3}{|c|}{ BFFS } \\
\hline & HR & $95 \% \mathrm{CI}$ & $P$-value & HR & $95 \% \mathrm{CI}$ & $P$-value \\
\hline Primary disease status & 4.49 & $1.41-14.34$ & 0.011 & 4.04 & $1.28-12.75$ & 0.017 \\
\hline \multicolumn{7}{|l|}{ (controlled vs. uncontrolled) } \\
\hline ECOG performance status & 3.87 & $1.79-8.36$ & 0.001 & 2.62 & $1.28-5.35$ & 0.008 \\
\hline \multicolumn{7}{|l|}{$(0-1$ vs. $1-2)$} \\
\hline Histological type & 1.88 & $0.85-4.16$ & 0.119 & 1.92 & $0.84-4.35$ & 0.121 \\
\hline \multicolumn{7}{|l|}{ (NSCLC vs. SCLC) } \\
\hline Extracranial metastases & 1.46 & $0.61-3.52$ & 0.395 & 1.79 & $0.75-4.30$ & 0.193 \\
\hline (absent vs. present) & & & & & & \\
\hline
\end{tabular}

Table 4. Dose schedules for SRS $(n=63)$

CyberKnife dose $\left(\mathrm{BED}_{10}\right) \quad$ Number of plans $(\%)$

Single fraction

16 Gy $(41.6 \mathrm{~Gy})$

$1(1.6)$ 


\begin{tabular}{|c|c|c|}
\hline 18 Gy (50.4 Gy) & 4 & $(6.3)$ \\
\hline 20 Gy (60 Gy) & 19 & $(30.2)$ \\
\hline 22 Gy (70.4 Gy) & 6 & $(9.5)$ \\
\hline 24 Gy (81.6 Gy) & 1 & $(1.6)$ \\
\hline \multicolumn{3}{|l|}{ Multiple fractions } \\
\hline $18 \mathrm{~Gy} / 3 \mathrm{fx}(28.8 \mathrm{~Gy})$ & 1 & $(1.6)$ \\
\hline $21 \mathrm{~Gy} / 3 \mathrm{fx}(35.7 \mathrm{~Gy})$ & 4 & $(6.3)$ \\
\hline $24 \mathrm{~Gy} / 3 \mathrm{fx}(43.2 \mathrm{~Gy})$ & 10 & $(15.9)$ \\
\hline 27 Gy/3 fx (51.3 Gy) & 7 & $(11.1)$ \\
\hline $28 \mathrm{~Gy} / 4 \mathrm{fx}(47.6 \mathrm{~Gy})$ & 1 & $(1.6)$ \\
\hline 30 Gy/3 fx (60 Gy) & 8 & $(12.7)$ \\
\hline 35 Gy/5 fx (59.5 Gy) & 1 & $(1.6)$ \\
\hline
\end{tabular}

SRS: stereotactic radiosurgery; $\mathrm{BED}_{10}$ : biologic equivalence dose for $\alpha / \beta=10$; Gy: gray, fx: fraction. 


\section{Figure Legends}

Figure 1. An example of the magnetic resonance imaging (MRI) response after CyberKnife (Accuray Inc., Snnyvale, CA, USA) based stereotactic radiosurgery (SRS). (A) Pre-SRS axial T1-weighted MRI with gadolinium illustrates a $2.5 \mathrm{~cm}$ sized enhancing mass on the Left side of the cerebellum. (B) PostSRS Axial T1-weighted MRI with gadolinium at 5 months shows a partial response in which the initial mass almost disappeared.

Figure 2. Overall survival (A) and intracranial progression free survival (B) of recursive partitioning analysis class II/III lung cancer patients with brain metastases.

Figure 3. Overall survival difference according to primary disease status (A) and extracranial metastases (B) for recursive partitioning analysis class II patients.

Figure 4. Overall survival difference according to primary disease status and (A) extracranial metastases (B) for recursive partitioning analysis class III patients. 